\title{
Joining Nanotubes Comprising Nucleobase-carrying Amphiphilic Polypeptides
}

\author{
Toru Itagaki ${ }^{a}$, Yuna Ueda ${ }^{a}$, Kenji Itabashib, Hirotaka Uji ${ }^{a}$, and Shunsaku Kimura ${ }^{\star a}$ \\ This paper is dedicated to the memory of Professor Robert Schwyzer, Mr. Peptide.
}

\begin{abstract}
Three kinds of amphiphilic polypeptides, $\mathrm{X}$-poly(sarcosine)- $b$-(L-Leu-Aib) ${ }_{6}(\mathrm{X}=$ adenine, thymine, glycolic acid), were synthesized and self-assembled in a tris buffer to take on nanotube morphology. The nanotubes were joined together to extend the nanotube length with the addition of trifluoroethanol and heat treatment at $50{ }^{\circ} \mathrm{C}$ for $24 \mathrm{~h}$. The length extension rate decreased in the order of adenine > glycolic acid > thymine depending on the $\mathrm{N}$-terminal chromophores. Adenine-adenine interactions between the nanotubes were found to be more prevalent upon joining the nanotubes than adenine-thymine interactions. Further, adenines on the nanotube surface could chelate with $\mathrm{Cu}(I)$ to thermodynamically stabilize the nanotube membrane. AFM imaging in liquid environment revealed that the membrane elasticity of the adenine nanotube was as high as ca. $1 \mathrm{MPa}$, which is considered to be strengthened as a result of the adenine-adenine interactions.
\end{abstract}

Keywords: Adenine · Amphiphilic Polypeptide · Molecular Assembly · Nanotube · Thymine

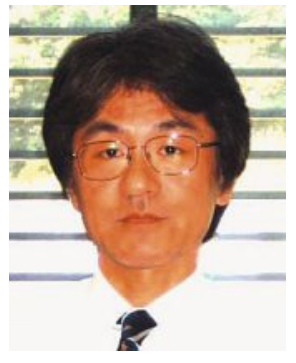

Shunsaku Kimura

was born in Kyoto, Japan in 1954. He received his BS (1976), MS (1978), and PhD (1982, Professor Y. Imanishi) degrees from Kyoto University. He joined the Department of Polymer Chemistry, Kyoto University, as Research Associate (1981), Lecturer (1992), and Associate Professor (1993). He moved to the Department of Material Chemistry, Kyoto University (1996), and in 1999 he was appointed Full Professor. He spent his postdoctoral career (1982-1984, 1986, Professor R. Schwyzer) at ETH-Zurich, Switzerland. He received the Award of the Society of Polymer Science, Japan, in 1999. His main interests are polymer supramolecular chemistry, peptide engineering, theranostic agents, immune-activating or suppressing materials, and optoelectronics devices.

${ }^{*}$ Correspondence: Prof. S. Kimura

E-mail: shun@scl.kyoto-u.ac.jp

aDepartment of Material Chemistry

Graduate School of Engineering, Kyoto University

Kyoto-Daigaku-Katsura, Nishikyo-ku

Kyoto 615-8510, Japan

bepartment of Polymer Chemistry,

Graduate School of Engineering, Kyoto University

Kyoto-Daigaku-Katsura, Nishikyo-ku

Kyoto 615-8510, Japan

\section{Introduction}

A variety of organic nanotubes have been constructed by various methods including via self-assembly, which is further categorized into four types according to the preparation pathways. ${ }^{[1]}$ This classification is based on the intermediate species during the molecular assembling processes; membrane-, nanoring-, stacking-, and supramolecular stacking components, which grow into the organic nanotubes. We have been studying membrane-based self-assembly of the organic nanotubes using amphiphilic polypeptides of poly(sarcosine)$b$-(L- or D-Leu-Aib) $(\mathrm{n}=6-8) \cdot{ }^{[2]}$ For example, poly(sarcosine)- $b$-(L-Leu-Aib) self-assembled into curved sheets just after injection into water, which converted to nanotubes upon heating at $90{ }^{\circ} \mathrm{C} .{ }^{[3]}$ The characteristic feature of the nanotube is the well-defined dimensions of $c a .80 \mathrm{~nm}$ diameter and ca. $250 \mathrm{~nm}$ length owing to the controlled size of the curved sheets prior to the formation of the nanotubes, which is a rare case in organic nanotubes. In light of the advantage of the well-defined dimensions of the nanotube, we aim to assemble the nanotubes into hierarchical structures by using the nanotube as a starting block.

Nucleobases have been used for supramolecular self-assembling by complementary pairing through hydrogen bonds. For example, nucleobase pairs were used for easy preparation of the supramolecular nucleoside phospholipids, [4] formation of cross-linking points in nanogels, ${ }^{[5]}$ and functionalization of supramolecular micelles. ${ }^{[6]}$ It is thus interesting to examine nanotubes with surfaces displaying nucleobases. Surface-modified nanotubes display two types of association modes; side-by-side and edge-to-edge. The former association is liable to make irregular aggregates of nanotubes, because the association regions between nanotubes may be variable from partial to full association and are out of control. On the other hand, edgeto-edge association will result in segregated nanotube structures after joining dissimilar nanotubes. The segregated nanotube has never been reported with a linear organic heterojunction by joining different semiconducting nanotube segments, however, stepwise nanotubular coassembly has been observed. ${ }^{[7]}$ The segregated nanotube was also obtained by using temperature-induced phase separation, ${ }^{[8]}$ but a method of joining two kinds of nanotubes showed limited success, ${ }^{[9]}$ and still remains to be explored. We therefore aim at extension of nanotube length by joining nanotubes via open edges with the help of base pairings. Our expectation is that the unfavorable side-by-side association between nanotubes for our hierarchical structure will be suppressed by the loss of the ability to form hydrogen bonds between nanotubes due to the HoogsteenType self-base pairing in the same nanotube membrane, ${ }^{[10]}$ when nucleobases are densely expressed on the nanotube surface. This suppressive effect of dense display of nucleobases on surface may be ascribed to a kind of molecular crowding effect.[11] 
We chose adenine as a nucleobase to modify the nanotube surface, because adenine has a high potential for hydrogen-bond formation as exemplified by the self-organized adenine ribbon. ${ }^{[12]}$ Adenine was therefore connected to the $N$-terminal of poly(sarcosine)- $b$-(L-LeuAib) (AL12, Fig. 1). The complementary nucleobase thymine was also connected to the $N$-terminal to obtain TL12 (Fig. 1), and glycolic acid was used to cap the $\mathrm{N}$ terminal to afford a reference compound (GL12, Fig. 1). The compositions of the amphiphilic polypeptides are summarized in Table 1, and the synthetic routes are shown in Scheme 1 (syntheses, NMR, and mass spectra in supporting information (SI); Scheme S1, Figs. S1-S6, www. Ingentaconnect.com, CHIMIA). In order to construct the hierarchical structure by joining the nanotubes, we have to solve the following problems; 1) suppression of the side-by-side association of nanotubes, 2) re-activation of the open mouths of nanotubes which became inert for sticking after nanotube preparation, and 3) suppression of lateral diffusion of the components in the nanotubular membrane to keep the two kinds of nanotubes to be joined from being phase-separated. The last issue is related with construction of phase-separated nanotubes along the long axis such as a nanotubular coassembly having a heterojunction. ${ }^{[7]}$ Further, successive joining of nanotubes will provide a small long channel comprising different compartments with individual inner wall surfaces, which are attractive from a view point of 'attoliter chemistry' as successive reaction fields. ${ }^{[13]}$ On the other hand, physicochemical properties of adenine and thymine, which have long been discussed, ${ }^{[14]}$ are expected to reveal new aspects when they are displayed densely on molecular assembly surfaces.

\section{Association of Nanotubes}

The reference compound GL12 was reported to self-assemble into curved sheets just after injection in water, which then converted to nanotubes upon heating at $90{ }^{\circ} \mathrm{C}$ for $10 \mathrm{~min} .{ }^{[3]}$ The nanotubes were dispersed well in water without any tendency of aggregation. With hydrophilic
Fig. 1. Molecular structures of amphiphilic block polypeptides having different groups at the $N$-terminals.

GL12<smiles>CCC(C)(NC(CC(C)C)C(=O)NC(C)(C)C(=O)C(C)(C)OC)C(C)(C)C(=O)CN(C)C(C)=O</smiles>

AL12<smiles>CCC(NC(C)(C)C(C)(C)C(=O)CN(C)C(=O)Cn1cnc2c(N)ncnc21)C(=O)NC(C)(C)C(=O)C(C)(C)OC</smiles>

TL12

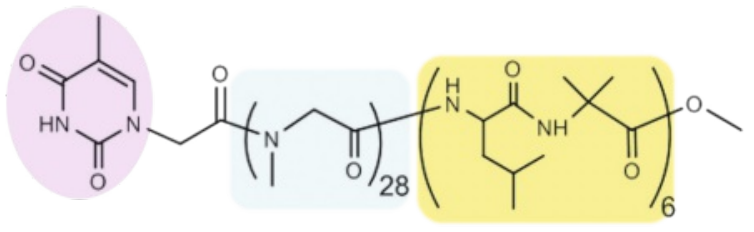

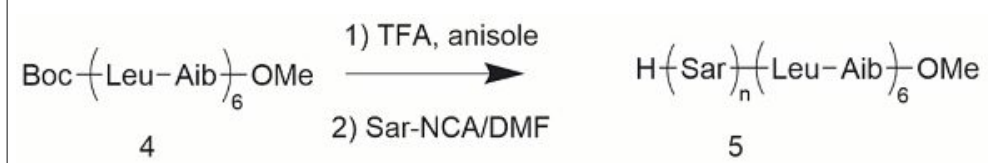

glycolic acid COMU,Oxyma,DIEA/DMF

5

$$
\mathrm{HO}-\mathrm{CH}_{2}-\underset{\mathrm{O}}{\mathrm{C}}(\mathrm{Sar})_{26}(\mathrm{Leu}-\mathrm{Aib})_{6} \mathrm{OMe}
$$

6

[GL12] $69 \%$

thymine-1-acetic acid COMU,Oxyma,DIEA/DMF

5

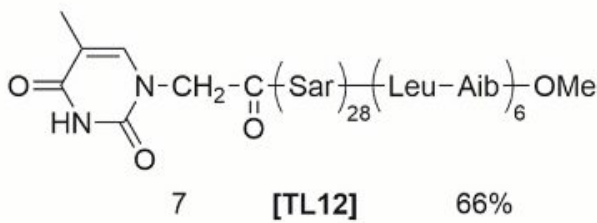

compound 3

COMU,Oxyma,DIEA/DMF

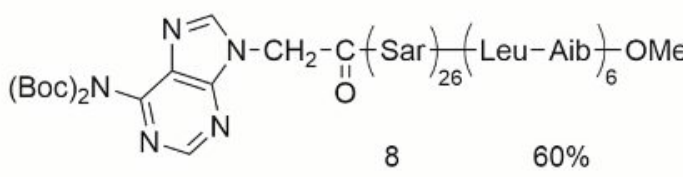

TFA, anisole

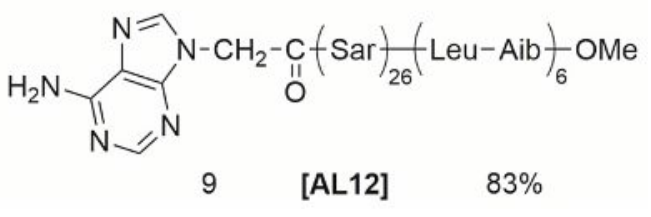

Scheme 1. Synthetic routes of GL12, TL12 and AL12.

Table 1. Compositions of GL12, TL12 and AL12

\begin{tabular}{|c|c|c|c|c|}
\hline & $\boldsymbol{N}$-terminal & $\boldsymbol{D P}_{\mathbf{n}}$ of poly(sarcosine) block & the helical hydrophobic block & $\boldsymbol{C}$-terminal \\
\hline GL12 & glycolic acid & 26 & -(L-Leu-Aib) ${ }_{6}^{-}$ & methyl ester \\
\hline TL12 & thymine-1-acetic acid & 28 & -(L-Leu-Aib) $)_{6}^{-}$ & methyl ester \\
\hline AL12 & adenin-9-yl-acetic acid & 26 & -(L-Leu-Aib)- & methyl ester \\
\hline
\end{tabular}


poly(sarcosine) chains covering densely the nanotube surfaces, non-specific association of nanotubes was avoided.

\subsection{Nanotubes Having Adenine and Thymine}

An aliquot of AL12 in ethanol was injected into a $10 \mathrm{mM}$ tris buffer $(\mathrm{pH} \mathrm{7.4)}$ containing $150 \mathrm{mM} \mathrm{NaCl}$ at $4{ }^{\circ} \mathrm{C}$ followed by heat treatment at $90{ }^{\circ} \mathrm{C}$ for $1 \mathrm{~h}$. TEM observations revealed formation of AL12 nanotubes having lengths much longer than the $c a .250 \mathrm{~nm}$ long GL12 nanotubes, accompanied by bundles and aggregations of the nanotubes (Fig. 2A). These aggregates should be formed by interactions between nanotubes. Adenine on the nanotube surface is therefore effective to associate nanotubes via both edge-to-edge and side-by-side. Since GL12 did not show any tendency of nanotube aggregation, the AL12 nanotube aggregation is ascribable to adenines on the nanotube surface, which should promote association via hydrogen bonding, hydrophobic interaction, and $\pi-\pi$ interaction. We therefore diluted AL12 by mixing with GL12. Accordingly, a mixture of AL12 and GL12 (1/1 mol/mol) afforded homogeneous and well-dispersed nanotubes with an average length of $c a .250 \mathrm{~nm}$ upon a heat treatment at $70{ }^{\circ} \mathrm{C}$ for $3 \mathrm{~h}$ after injection in a tris buffer (A50, Fig. 2B, C). In the TEM images, some nanotubes contacted with each other, but these contacts were as a result of the concentration effect of the solution on the TEM grid, and were not due to secondary interactions between nanotubes.

An aliquot of TL12 in ethanol was similarly injected into a tris buffer. With heat treatment at $90{ }^{\circ} \mathrm{C}$ for $1 \mathrm{~h}$, well-dispersed nanotubes were obtained in this case but with a wide distribution of the nanotube lengths up to over $1 \mu \mathrm{m}$ length (Fig. 3A). The wide distribution became narrower upon mixing in GL12 at a molar ratio of $1 / 1$ and with a heat treatment at $90{ }^{\circ} \mathrm{C}$ for $1 \mathrm{~h}$ (T50, Fig. 3B, C). Thymine on the nanotube surface therefore only causes the nanotubes to associate edge-to-edge. Taken together, adenine and thymine on the nanotube surfaces are effective for promoting association of the nanotubes but to different extents. Adenine seems to possess a tendency to promote molecular association more than thymine, and nanotube elongation through edge-to-edge association occurs under more moderate conditions than the side-by-side association.

\subsection{Nanotube Stability at $50^{\circ} \mathrm{C}$ for $24 \mathrm{~h}$}

A50, T50, or GL12 nanotubes (G100) were prepared by heat treatments of $70{ }^{\circ} \mathrm{C}$ for $3 \mathrm{~h}, 90{ }^{\circ} \mathrm{C}$ for $1 \mathrm{~h}$, and $90{ }^{\circ} \mathrm{C}$ for $10 \mathrm{~min}$, respectively, followed by examination of nanotube stability at $50{ }^{\circ} \mathrm{C}$ for $24 \mathrm{~h}$. A50, T50, or GL12 nanotubes (G100) showed very similar histograms of nanotube lengths before and after heat treatment at $50{ }^{\circ} \mathrm{C}$ for $24 \mathrm{~h}$ (Fig. $4 \mathrm{~A}, \mathrm{~B}$, $\mathrm{C})$. The open mouths of the nanotubes are therefore inert for self-sticking at $50{ }^{\circ} \mathrm{C}$. Unexpectedly, a mixture of A50 and T50 also remained unchanged on the nanotube length histogram after heat treatment at $50{ }^{\circ} \mathrm{C}$ for $24 \mathrm{~h}$ (Fig. 4D). The adenine and thymine moieties displayed on the surfaces of the nanotubes, which were prepared with heating at $70{ }^{\circ} \mathrm{C}$ and $90{ }^{\circ} \mathrm{C}$, respectively, therefore lost association ability
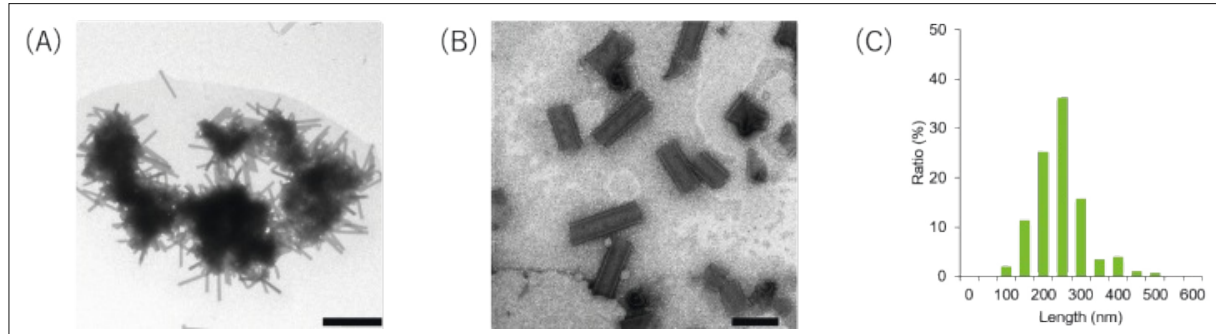

Fig. 2. TEM images of $A L 12(A)$ and a mixture of $A L 12$ and GL12 at a molar ratio of $1 / 1 \mathrm{~mol} / \mathrm{mol}$ (A50) (B). Bars $=2 \mu \mathrm{m}(A)$ and $200 \mathrm{~nm}(B)$. The histogram of the nanotube length of $A 50(C)$.

(A)

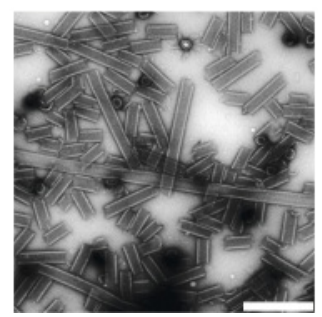

(B)

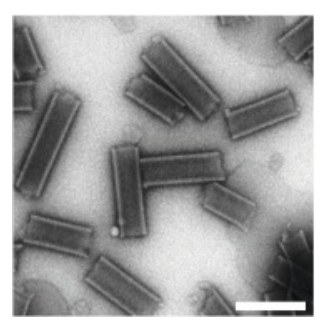

(C)

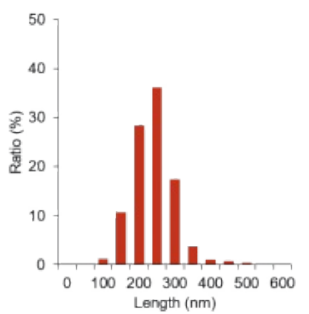

Fig. 3. TEM images of TL12 nanotubes (A) and a mixture of TL12 and GL12 at a molar ratio of 1/1 $\mathrm{mol} / \mathrm{mol}(\mathrm{T50})(B)$. Bars $=500 \mathrm{~nm}(A)$ and $200 \mathrm{~nm}(B)$. The histogram of the nanotube lengths of $\mathrm{T} 50$ (C).

through complementary hydrogen-bond formation between adenine and thymine moieties. It is considered that the adenine and thymine moieties should be stabilized by respective self-association on the nanotube surfaces, which makes it impossible to associate the adenine moieties of one nanotube with the thymine moieties of the other nanotube.

\subsection{Nanotube Elongation with Help of Trifluoroethanol}

The A50, T50, and G100 nanotubes were incubated at $50{ }^{\circ} \mathrm{C}$ for $24 \mathrm{~h}$ in the presence of trifluoroethanol of varying concentrations $1 \%, 3 \%$, and $5 \%$. All the nanotubes in the presence of $5 \%$ trifluoroethanol extended the nanotube length by nearly ten-times with a wider length distribution than the initial state (Fig. 5). The extension rate in the presence of $3 \%$ TFE is dependent on the chemical species at the $N$-terminal groups of the hydrophilic poly(sarcosine) blocks as the elongation effect of trifluoroethanol was the largest on A50 (Fig. 5A) and the smallest on T50 (Fig. 5B) in the order of adenine $>$ glycol acid $>$ thymine.

Nanotube elongation in the presence of $3 \%$ trifluoroethanol was further studied by varying the mixing ratios of T50 and G100 in A50 (Fig. 6) to examine the additive effects of T50 and G100 on the nanotube elongation of A50. The nanotubes extended the lengths in the presence of $20 \%$ or $50 \%$ G100 similarly to $100 \%$ A50 (Fig. 6A, B, C), but the length extension became moderate by the excess addition of $80 \%$ G100 (Fig. 6A, D). On the other hand, the length extension of A50 was severely suppressed by the presence of $20 \%$ T50 (Fig. 6A, B) and more (Fig. $6 \mathrm{~A}, \mathrm{C}, \mathrm{D})$. These results also support the interpretation that the ability to induce the nanotube elongation decreases in the order of adenine $>$ glycol acid $>$ thymine at the $\mathrm{N}$ terminal. Trifluoroethanol is well known as a good solvent for the hydrophobic block of (L-Leu-Aib) ${ }_{6}$ to maintain helical structures. It is therefore considered that trifluoroethanol infiltrates the hydrophobic core of the nanotube membrane to loosen the ordered state of the nanotube membrane into an amorphous adhesive state especially at the edge of the nanotube, which should allow the nanotubes to stick together. In other words, trifluoroethanol can re-activate the open mouths of the nanotubes for nanotube sticking. An additional effect of adenine on nanotube elongation owing to the favorable self-association property can explain the strong ability of A50 for nanotube elongation. On the other hand, thymine seems to have a suppressive effect on nanotube elongation triggered by the addition of trifluoroethanol. 


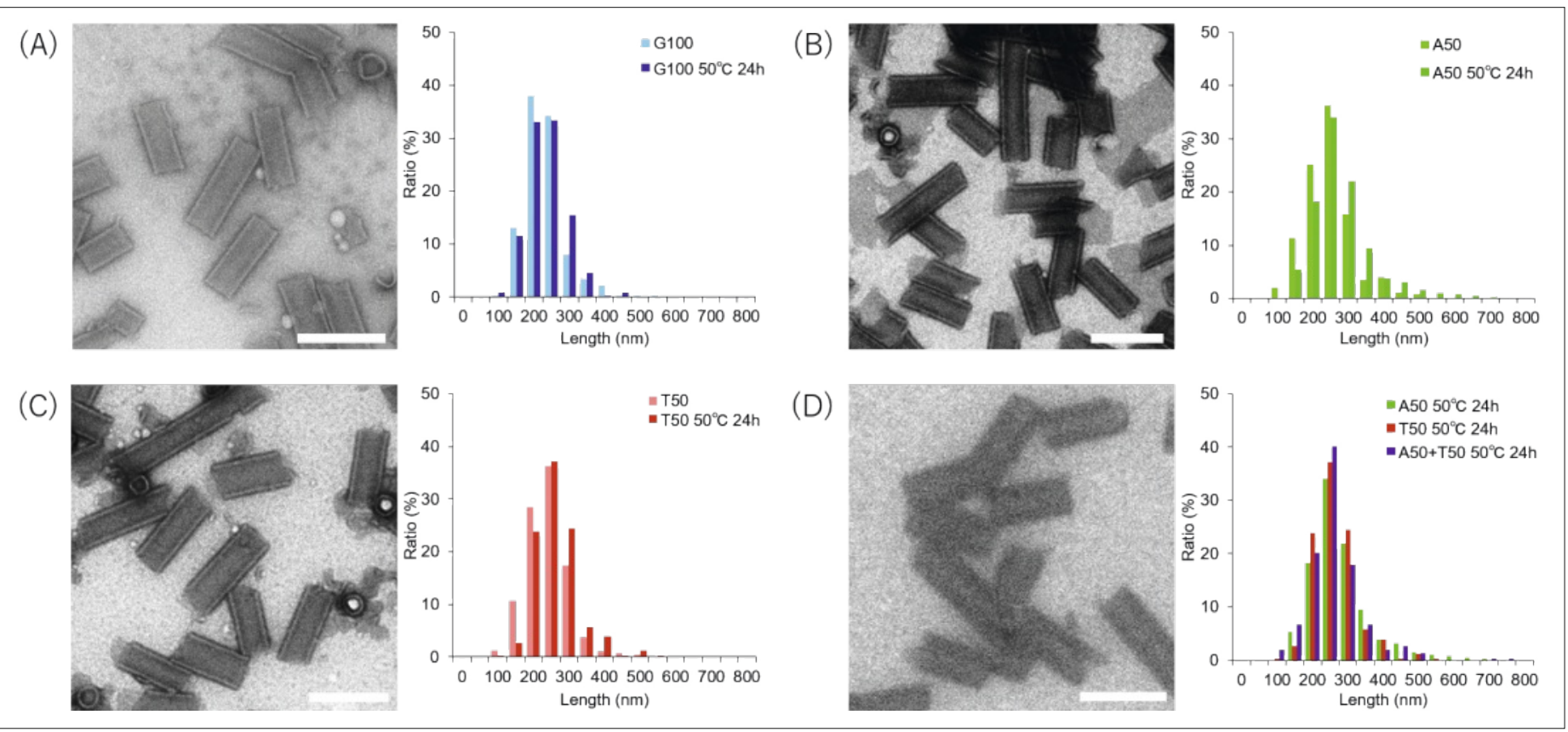

Fig. 4. TEM images with negative staining (left) of G100 (A), A50 (B), T50 (C), and a mixture of $A 50$ and T50 (D) after incubation at $50{ }^{\circ} \mathrm{C}$ for $24 \mathrm{~h}$. Bars $=250 \mathrm{~nm}$. Histograms of the nanotube lengths are before and after incubation at $50^{\circ} \mathrm{C}$ for $24 \mathrm{~h}(\mathrm{~A}, \mathrm{~B}, \mathrm{C})$. Histograms of A50, T50 and a mixture of $\mathrm{A} 50$ and T50 after incubation at $50^{\circ} \mathrm{C}$ for $24 \mathrm{~h}(\mathrm{D})$.

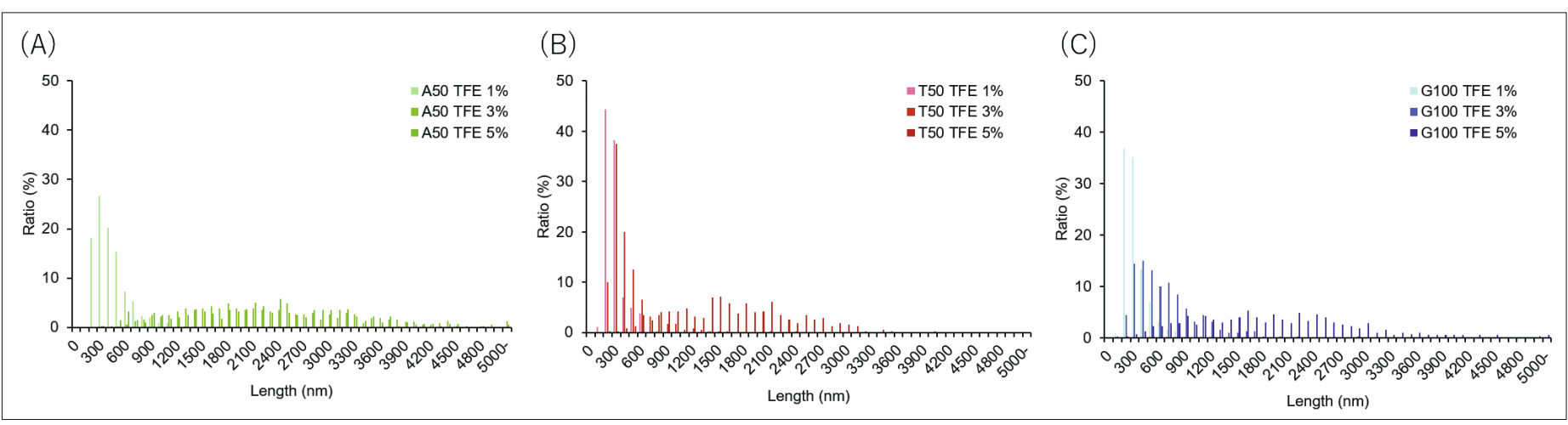

Fig. 5. Histograms of the nanotube lengths with heat treatment at $50{ }^{\circ} \mathrm{C}$ for $24 \mathrm{~h}$ in the presence of trifluoroethanol of varying concentrations from $1 \%, 3 \%$ and $5 \%$. A50 (A), T50 (B), and G100 (C).

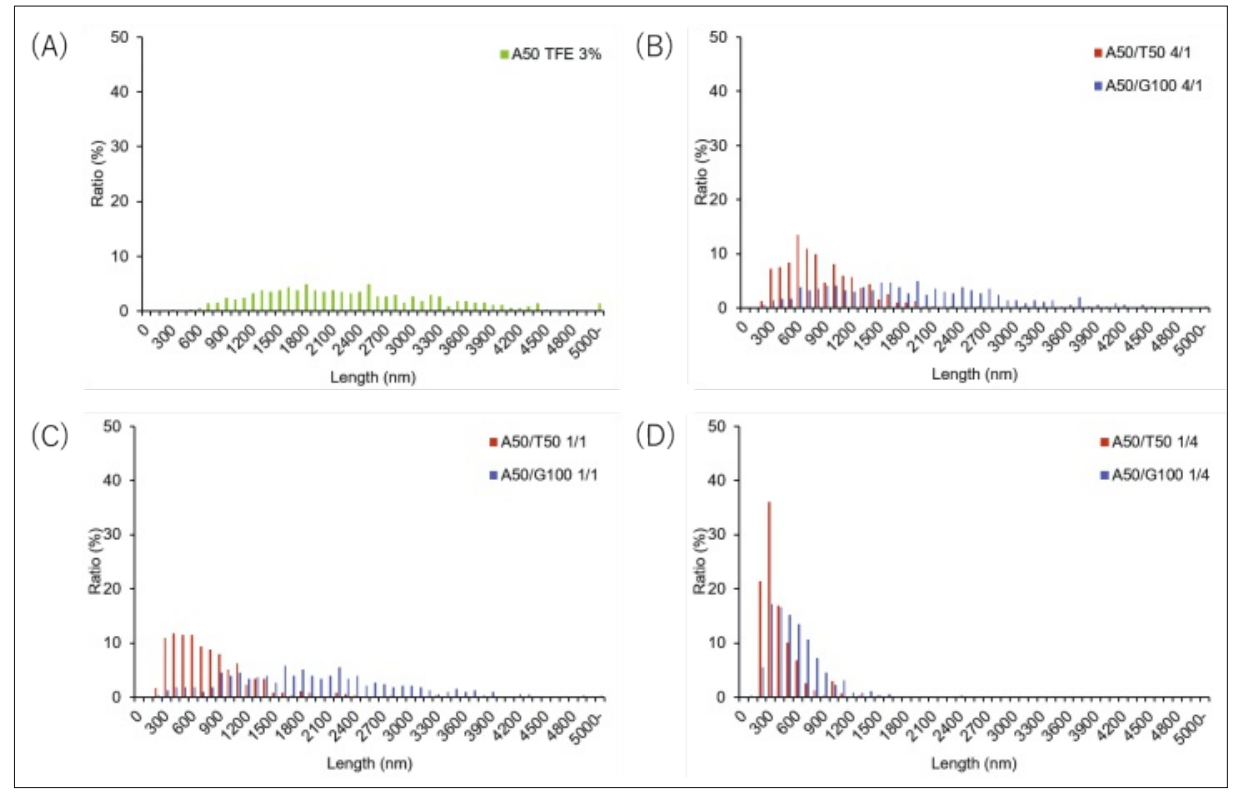

Fig. 6. Histograms of the nanotube lengths after incubation at $50{ }^{\circ} \mathrm{C}$ for $24 \mathrm{~h}$ in the presence of $3 \%$ trifluoroethanol. A50 (A). Mixtures of A50 and T50 (red) and A50 and G100 (blue) at 4/1 mol/ $\mathrm{mol}(\mathrm{B}), 1 / 1 \mathrm{~mol} / \mathrm{mol}(\mathrm{C})$, and $1 / 4 \mathrm{~mol} / \mathrm{mol}(\mathrm{D})$.

\subsection{The Effect of AMP or TMP} Addition on Nanotube Elongation

The adenine moieties displayed on the nanotube surface promoted elongation with the addition of trifluoroethanol. The effect of adenine monophosphate (AMP) addition was examined on nanotube elongation of T50 (Fig. 7A). The histograms of the nanotube lengths clearly indicate the additive effect of AMP on T50 elongation in the presence of $3 \%$ trifluoroethanol, which is similar to the additive effect of A50 (Fig. 7B). On the other hand, thymine monophosphate (TMP) did not show a suppressive effect on A50 elongation in the presence of $3 \%$ trifluoroethanol (Fig. 7C), which is in contrast with the suppressive effect of T50 (Fig. 7D). The suppressive effect of the thymine moieties on the nanotube elongation is therefore interpreted as a consequence of multivalent interactions of the thymine moieties densely displayed on the nanotube surface. 


\section{Adenine Nanotubes}

The nanotubes displaying adenine on the surface were able to elongate nanotube length owing specifically to the self-association property of adenine. Adenine-adenine interactions have long been discussed in terms of dispersion force, photo-excitation, and charge transfer, etc. ${ }^{[15]} \mathrm{A} 50$ is an attractive system to examine adenine-adenine interactions from a different point of view from the dense display on two-dimensional membranes. The characteristic features of A50 were therefore analyzed in depth.

\subsection{Copper(II)-Adenine Coordination}

The purine ligand of adenine was reported to build dinuclear complexes with $\mathrm{Cu}(\mathrm{II}) .{ }^{[16]}$ An aliquot of AL12, GL12, and $\mathrm{CuBr}_{2}$ in ethanol was injected into a tris buffer followed by heat treatment at $70{ }^{\circ} \mathrm{C}$ for $3 \mathrm{~h}$ to afford copper-chelated nanotubes (Cu-A50). A50 was separately prepared and mixed with $\mathrm{Cu}-\mathrm{A} 50$. A mixture of $\mathrm{A} 50$ and $\mathrm{Cu}-\mathrm{A} 50$ was observed by TEM without staining (Fig. 8A, Fig. S7 in SI). Indeed, there are two types of nanotubes of dilute and dense colors, which are assignable respectively to $\mathrm{A} 50$ and $\mathrm{Cu}-\mathrm{A} 50$. The $\mathrm{Cu}-\mathrm{A} 50$ nanotube was imaged by the dense color over the whole surface of the nanotube, suggesting that the adenine moieties should be homogeneously distributed over the membranes comprising AL12 and GL12, and the chelation of two adenines with a $\mathrm{Cu}$ (II) also occurred homogeneously. Histograms of the nanotube lengths showed that the average length became longer with $\mathrm{Cu}$ (II) chelation (Fig. 8B). The nanotube membranes are therefore considered to be thermodynamically stabilized by the chelation.

The mixture was incubated at $50{ }^{\circ} \mathrm{C}$ for $3 \mathrm{~h}$ and $24 \mathrm{~h}$ in the presence of $3 \%$ trifluoroethanol. The histograms of the nanotube lengths revealed the length extension with time (Fig. 8B). The TEM image after $3 \mathrm{~h}$ incubation showed some nanotubes with dilute and dense colors, which are ascribable to the elongated nanotubes just after joining A50 with $\mathrm{Cu}-\mathrm{A} 50$. With further incubation for $24 \mathrm{~h}$, the nanotube images became monotone in the color, suggesting that the components of $\mathrm{A} 50$ and $\mathrm{Cu}-\mathrm{A} 50$ mixed together in the nanotubular membrane by lateral diffusion and/or exchange processes of $\mathrm{Cu}$ (II) from one site to the other (Fig. 8A). We currently speculate that the latter process is more plausible because the lateral diffusion of the components is considered to be less active at $50^{\circ} \mathrm{C}$.

\subsection{AFM Observation of Nanotubes}

A50, T50 and G100 were subjected to liquid AFM observations using a silicon substrate with surface modification by 3-aminopropyltriethoxysilane. An AFM image in liquid environment of the elongated nanotubes successfully observed A50 (Fig. 9A) with a height profile of $c a$. $70 \mathrm{~nm}$ (Fig. 9B). On the other hand, T50 and G100 were unsuccessfully imaged under the same conditions (Fig. S8 in SI). One reason for the failure to image T50 and G100 is the weak interaction of the nanotube surface with a silicon substrate covered with a thin layer of 3-aminopropyltriethoxysilane, resulting in no remaining nanotubes on the substrate because nanotubes on a substrate should be wiped out during scanning the AFM tip. The adsorption of A50 to a surface-modified silicon substrate therefore just fits to the AFM observation in light of nanotubes staying on the surface during scanning of the AFM tip, but the adsorption force is not strong enough to open the nanotube structure. Sometimes we observed flat sheets in the AFM images, which should be a result of opening up of the nanotube structure, because of strong interaction between the nanotube and substrate.

The membrane elasticity of A50 was evaluated by the nano-indentation method using liquid AFM. The elastic modulus value was found to be $c a$. $1 \mathrm{MPa}$ (Fig. S9 in

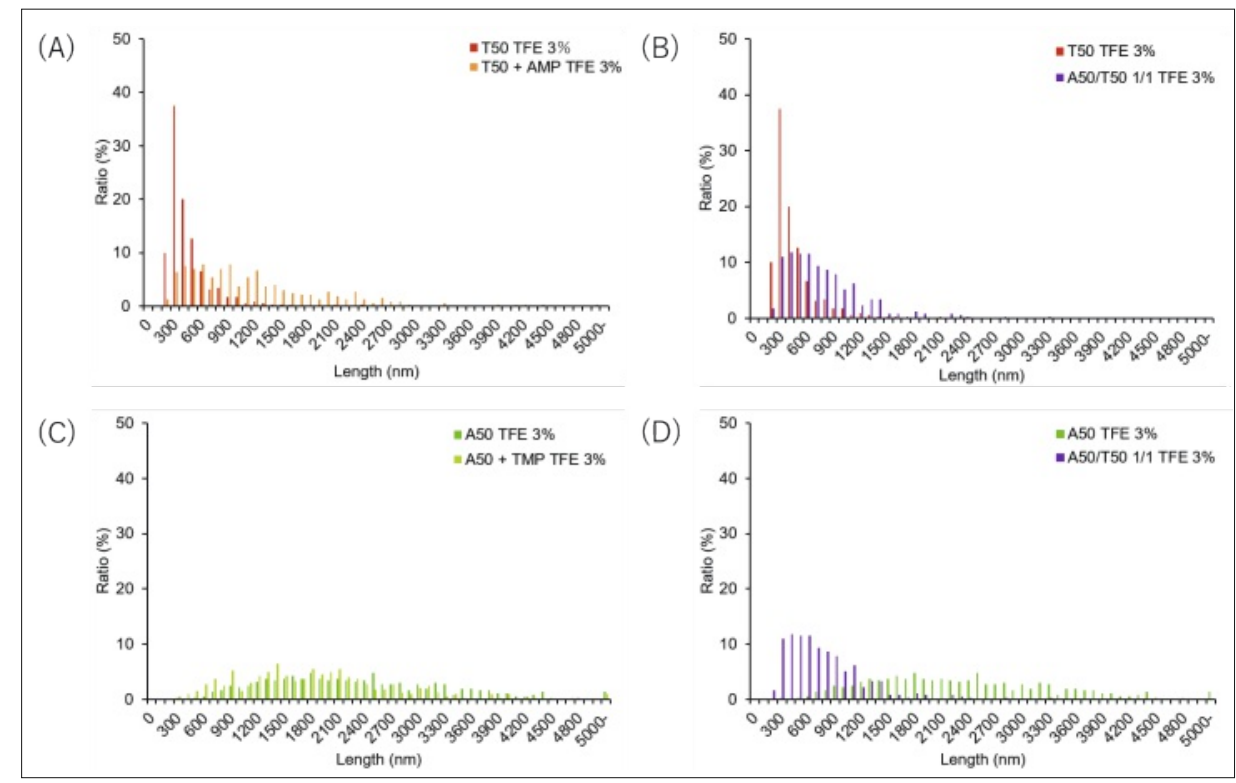

Fig. 7. Histograms of the nanotube lengths after incubation at $50{ }^{\circ} \mathrm{C}$ for $24 \mathrm{~h}$ in the presence of $3 \%$ trifluoroethanol. T50 and a mixture of T50 and AMP (A). T50 and a mixture of T50 and A50 (B). A50 and a mixture of A50 and TMP (C). A50 and a mixture of A50 and T50 (D).
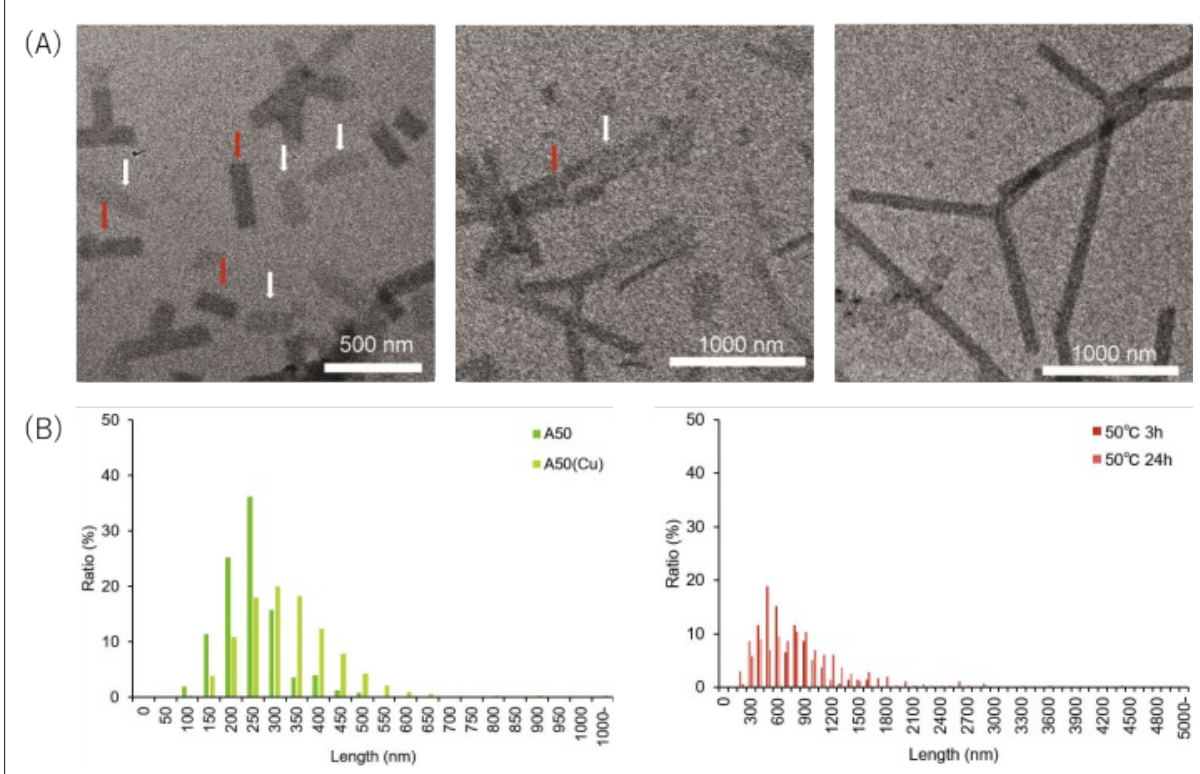

Fig. 8. (A) TEM images of a mixture of A50 and Cu-A50 without staining just after mixing (left panel; red arrows indicate the dense-colored nanotubes and white arrows the dilute-colored nanotubes), after $3 \mathrm{~h}$ incubation at $50^{\circ} \mathrm{C}$ (middle panel; the red and white arrows indicate the same as the left panel), and after $24 \mathrm{~h}$ incubation at $50^{\circ} \mathrm{C}$. (B) Nanotube length histogram of a A50 and Cu-A50. 
SI), which is lower than the vesicles comprising similar amphiphilic polypeptides showing 4-12 MPa (Fig. 9C). ${ }^{[17]}$ However, taking into a consideration the structural difference between the closed inner space of vesicles and the open space of nanotubes, the membrane elasticity measured here will be intrinsically far smaller with nanotubes than vesicles. A50 nanotube membrane showing moderate membrane elasticity therefore should be strengthened by adenine self-association on the surface.

The plasticity indices of A50 were nearly in the range from 0.3 to 0.6 (Fig. 9D, Fig. S9 D in SI), which are similar to those of vesicles.[17] A50 is therefore considered to be similarly durable to the vesicles, which may be a striking aspect of A50 despite the fragile nanotube open structure.

\section{Conclusions}

Nucleobases are well known to act as recognition units for nucleic acids. A base pairing between adenine and thymine via two hydrogen bonds is a typical example. However, there are various other types of base pairings categorized into the Hoogsteen type, the wobble type, and widely observed non-Watson-Crick type. The attractive interactions between nucleobases arise from hydrogen bonding, dispersion forces, hydrophobic interactions, and $\pi-\pi$ stacking. These elements depend on the surrounding environment to occur, resulting in environment-dependent base pairings. In the present case, adenine and thymine were densely displayed on nanotubes. When the adenine density was ca. 1 adenine $/ 2 \mathrm{~nm}^{2}$ (nanotubes prepared from $100 \%$ AL12), inter-nanotube sideby-side and edge-to-edge interactions via self-base pairing resulted in nanotube aggregates. With dilution of adenine density down to $c a$. 1 adenine/4.5 $\mathrm{nm}^{2}$ (nanotubes prepared from an equal mixture of AL12 and GL12), inter-nanotube edge-to-edge interaction was the sole association effect on joining nanotubes. This difference is simply explained by easier crosslinking of two adenines on the same nanotube membrane after joining (edge-to-edge interaction) than the different nanotube membranes (side-by-side interaction). On the other hand, thymines were ineffective for inter-nanotube associations, even though the counterpart of the thymine-displaying nanotube was the adenine-displaying nanotube. It is therefore considered that the complementary interaction between adenine and thymine becomes insignificant when they are displayed on molecular as-

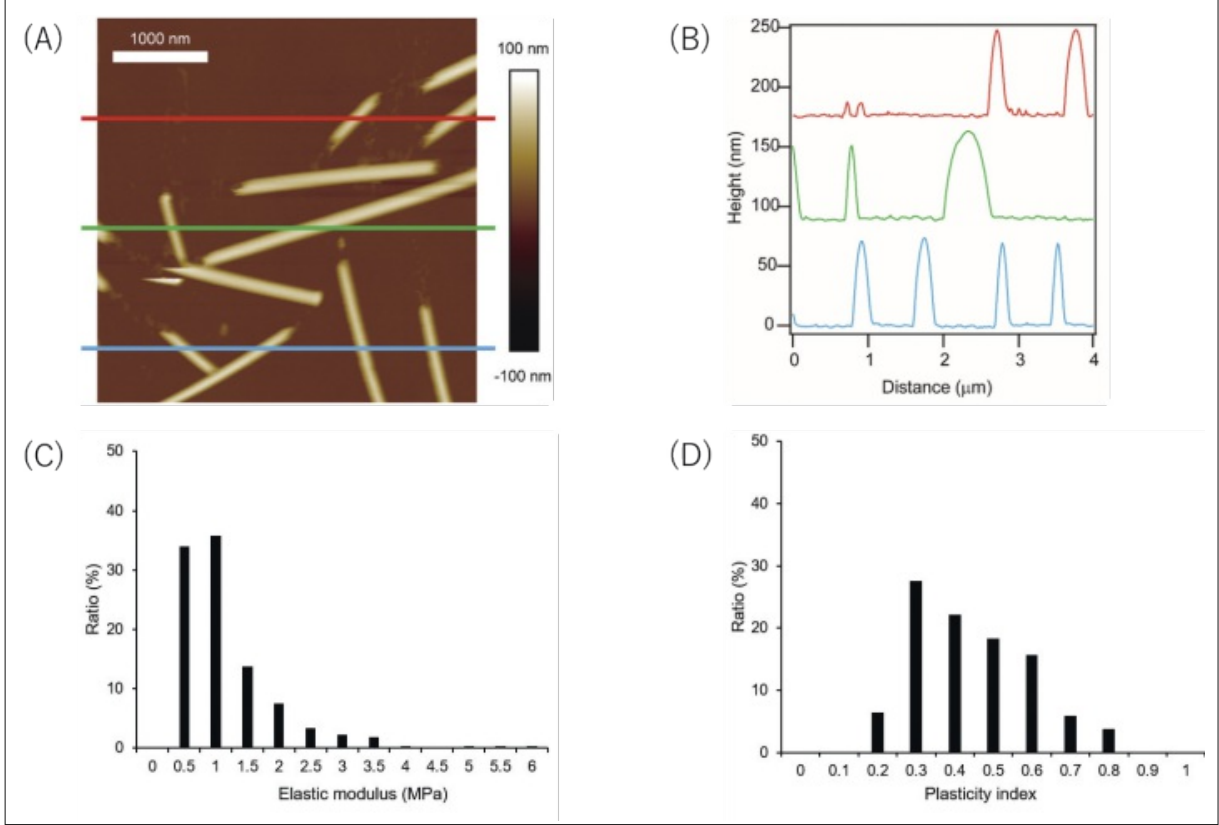

Fig. 9. (A) An AFM image in liquid environment of $A 50$ after incubation at $50{ }^{\circ} \mathrm{C}$ for $24 \mathrm{~h}$ in the presence of $3 \%$ trifluoroethanol. The medium is a tris buffer $(0.01 \mathrm{M}, \mathrm{pH} 7.4)$ containing $0.1 \mathrm{M}$ $\mathrm{NaCl}$. A silicon substrate with surface modification by 3-aminopropyltriethoxysilane was used. (B) Height profiles of A50 nanotubes. (C) Elastic modulus distribution of A50 nanotube calculated by the Hertzian contact model. (D) Histogram of plasticity index of A50 nanotube.

semblies in water. Interestingly, adenineadenine interaction is found to be the most prevailing interaction in the case of the adenine-displaying nanotubes.

\section{Acknowledgements}

This research was supported partially by JSPS KAKENHI Grant Number JP16H02279.

Received: June 19, 2018

[1] T. Shimizu, B Chem. Soc. Jpn. 2018, 91, 623, DOI: $10.1246 / \mathrm{bcsj} .20170424$

[2] a) M. Ueda, A. Makino, T. Imai, J. Sugiyama, S. Kimura, J. Pept. Sci. 2011, 17, 94, DOI: 10.1002/ psc.1304; b) M. Ueda, A. Makino, T. Imai, J. Sugiyama, S. Kimura, Soft Matter 2011, 7, 4143, DOI: 10.1039/c0sm01308k; c) M. Ueda, A. Makino, T. Imai, J. Sugiyama, S. Kimura, Chem. Commun. 2011, 47, 3204, DOI: 10.1039/ c0cc04209a; d) A. Uesaka, M. Ueda, A. Makino, T. Imai, J. Sugiyama, S. Kimura, Langmuir 2014, 30, 1022, DOI: 10.1021/la404784e.

[3] T. Kanzaki, Y. Horikawa, A. Makino, J. Sugiyama, S. Kimura, Macromol. Biosci. 2008, 8, 1026, DOI: 10.1002/mabi.200800071.

[4] D. L. Wang, C. L. Tu, Y. Su, C. Zhang, U. Greiser, X. Y. Zhu, D. Y. Yan, W. X. Wang, Chem. Sci. 2015, 6, 5090, DOI: 10.1039/ c5sc90038g.

[5] M. Fan, J. X. Yan, H. P. Tan, Y. T. Miao, X. H. Hu, J. Mater. Chem. B 2014, 2, 8399, DOI: 10.1039/c4tb01106f.

[6] C. C. Cheng, I. H. Lin, J. K. Chen, Z. S. Liao, J. J. Huang, D. J. Lee, Z. Xin, Macromol. Biosci. 2016, 16, 1415, DOI: 10.1002/mabi.201600189.

[7] W. Zhang, W. S. Jin, T. Fukushima, A. Saeki, S. Seki, T. Aida, Science 2011, 334, 340, DOI: 10.1126/science. 1210369
[8] T. Hattori, T. Itagaki, H. Uji, S. Kimura, J. Phys. Chem. B 2018, 122, 7178, DOI: 10.1021/acs. jpcb.8b03419.

[9] T. Itagaki, S. Kurauchi, T. Uebayashi, H. Uji, S. Kimura, ACS Omega 2018, 3, 7158, DOI: 10.1021/acsomega.8b01073.

[10] a) N. B. Leontis, J. Stombaugh, E. Westhof, Nucleic Acids Res. 2002, 30, 3497, DOI: 10.1093/ nar/gkf481; b) L. Qi, L. L. Gundersen, E. J. Chamgordani, C. H. Gorbitz, Crystengcomm. 2016, 18, 6352, DOI: 10.1039/c6ce01159d.

[11] D. Miyoshi, N. Sugimoto, Biochimie 2008, 90 1040, DOI: 10.1016/j.biochi.2008.02.009.

[12] a) D. Dobrzynska, L. B. Jerzykiewicz, J. Am. Chem. Soc. 2004, 126, 11118, DOI: 10.1021/ ja0470113; b) W. Zierkiewicz, D. Michalska, P. Hobza, Phys. Chem. Chem. Phys. 2010, 12, 2888, DOI: $10.1039 / \mathrm{b} 920433 \mathrm{~d}$

[13] T. Shimizu, J. Polym. Sci. Polym. Chem. 2008 46, 2601, DOI: 10.1002/pola.22652.

[14] a) Y. L. Li, M. C. Maurel, C. Ebel, J. Vergne, V. Pipich, G. Zaccai, Eur. Biophys. J. Biophys. 2008, 37, 173, DOI: 10.1007/s00249-007-02138; b) J. M. Carnerero, A. Sanchez-Coronilla, E. I. Martin, A. Jimenez-Ruiz, R. Prado-Gotor, Phys. Chem. Chem. Phys. 2017, 19, 22121, DOI: $10.1039 / \mathrm{c} 7 \mathrm{cp} 03692 \mathrm{~b}$

[15] a) M. Aida, C. Nagata, Chem. Phys. Lett. 1982 86, 44, DOI: 10.1016/0009-2614(82)83113 8; b) A. Banyasz, T. Gustavsson, D. Onidas, P. Changenet-Barret, D. Markovitsi, R. Improta, Chem.- Eur. J. 2013, 19, 3762, DOI: 10.1002/ chem.201202741; c) V. G. Stavros, J. R. R. Verlet, Annu. Rev. Phys. Chem. 2016, 67, 211, DOI: 10.1146/annurev-physchem-040215-112428.

[16] J. Thomas-Gipson, G. Beobide, O. Castillo, M. Froba, F. Hoffmann, A. Luque, S. Perez-Yanez, P. Roman, Cryst. Growth. Des. 2014, 14, 4019 , DOI: $10.1021 / \operatorname{cg} 500634 \mathrm{y}$.

[17] C. J. Kim, M. Ueda, T. Imai, J. Sugiyama, S. Kimura, Langmuir 2017, 33, 5423, DOI: 10.1021/acs.langmuir.7b00289. 